\title{
CATKey: Customizable and Adaptable Touchscreen Keyboard with Bubble Cursor-Like Visual Feedback
}

\author{
Kentaro Go ${ }^{1}$ and Yuki Endo ${ }^{2}$ \\ ${ }^{1}$ Interdisciplinary Graduate School of Medicine and Engineering, University of Yamanashi \\ 4-3-11 Takeda, Kofu 400-8511 Japan \\ go@yamanashi.ac.jp \\ ${ }^{2}$ Yamanashi R\&D Center, HAL Laboratory, Inc. \\ 1999-9 Ryuoshinmachi, Kai 400-0111 Japan \\ endo@golab.org
}

\begin{abstract}
This paper describes our ongoing project related to touchscreen keyboard interfaces. This customizable and adaptable touchscreen keyboard with bubble cursor-like visual feedback, CATKey, is a software keyboard for touchscreens that is designed to provide adaptable and customizable functions. We discuss its concept, prototype, and tentative evaluation.
\end{abstract}

Keywords: adaptation, bubble cursor, customization, touchscreen keyboard.

\section{Introduction}

Touchscreen interfaces have been attracting attention in recent years because their functionality and usability for mobile phones and information appliances have become better known. Recently, they are used as mobile phone interfaces for iPhone by Apple, KE850 by LG, and FOMA D800iDS by Mitsubishi. They are used also in public-use terminals including automated teller machines, ticket machines, and information kiosks. Touchscreen keyboards are a fundamental application for touchscreen interfaces and are used in several application areas. The key arrangement for touchscreen keyboards typically uses the standard Qwerty arrangement because users can transfer their skills and experience gained using common hardware keyboards [5]. Previous studies have shown that the text entry rate for a touchscreen keyboard is considerably slower than that for hardware Qwerty keyboard, even for expert typists [6, 7].

This paper reports our ongoing efforts at designing a Customizable and Adaptable Touchscreen Keyboard with bubble cursor-like visual feedback (CATKey). We are particularly interested in involving a wide range of user classes by improving perceived usability without decreasing the text entry rate. Many touchscreen systems are targeted at the public-use market. For that reason, users' skills and experiences are varied. Users would refuse to use them if the text entry method were to provide low perceived usability. 


\section{Touchscreen Keyboard Design}

Text entry tasks with touchscreen keyboards are cumbersome because (1) the user cannot touch, feel, or distinguish the positions of the keys displayed on the screen; and (2) it is difficult to feel the visual and tactile feedback from the keys, especially with conventional touchscreen devices ${ }^{1}$. However, touchscreen keyboards have practical advantages because (1) they can be designed as customizable and adaptable keyboards; and (2) they can be designed to display the visual feedback of and on the keys.

Our approach to designing touchscreen keyboards is to address the weak points using customizable and adaptable functions and effective visual feedback to improve their perceived usability.

\subsection{Customizable Key Layout}

Figure 1 shows an overview of our keyboard design. The left panel is the initial layout of keys. Each key has a maximum area. Consequently, the overall key area forms a Voronoi diagram. The right panel presents a key layout customized by a user, which is done in the customization mode of CATKey. The customized key area also forms a Voronoi diagram.
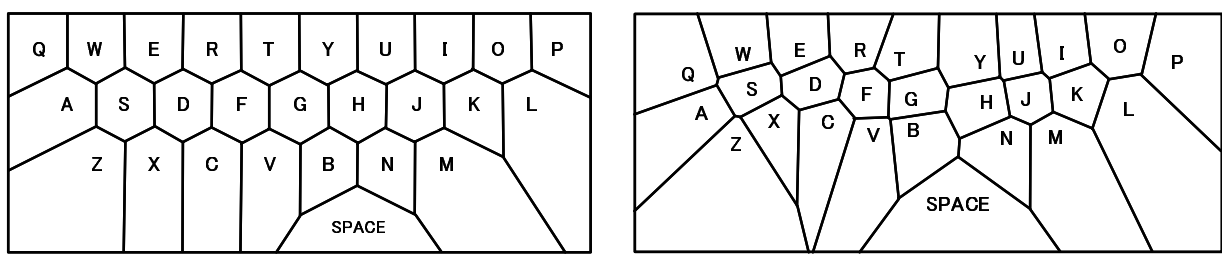

Fig. 1. Overview of CATKey: customizable and adaptable touchscreen keyboard with bubble cursor-like visual feedback. Left: the initial layout of keys. Right: a key layout customized by the user.

\subsection{Adaptable Keys}

We designed an adaptable function similar to [3]. In the adaptive mode of CATKey, the key centroid is moved to the centroid of recorded keystroke points in each key (Fig. 2). The keystroke coordinates are recorded for the period of the pre-specified number of keystrokes.

\subsection{Visual Feedback}

Figure 3 depicts the design of visual feedback in CATKey. The left panel

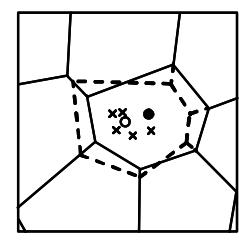

Fig. 2. The key adaptation mechanism o CATKey. Black point: the original centroid $\mathrm{X}$-mark: keystroke points. White circle: th centroid of the keystroke points and th weighted original centroid. Dotted line recalculated key area.

${ }^{1}$ Although some studies of tactile displays (tactile VDTs) have emerged [1], most touchscreen display products do not have tactile feedback. 
shows an actual view of CATKey. We assigned a round key area to communicate the touched location to the user. The yellow $\mathrm{f}$ and $\mathrm{j}$ keys show the home positions of index fingers. The presence of the line and color of keys can be modified using the parameter-setting dialogue. The right panel shows the bubble cursor-like visual feedback [2]. The bubble-shape cursor in red adjusts dynamically to the closest key and provides the sensation of an offset cursor [8].
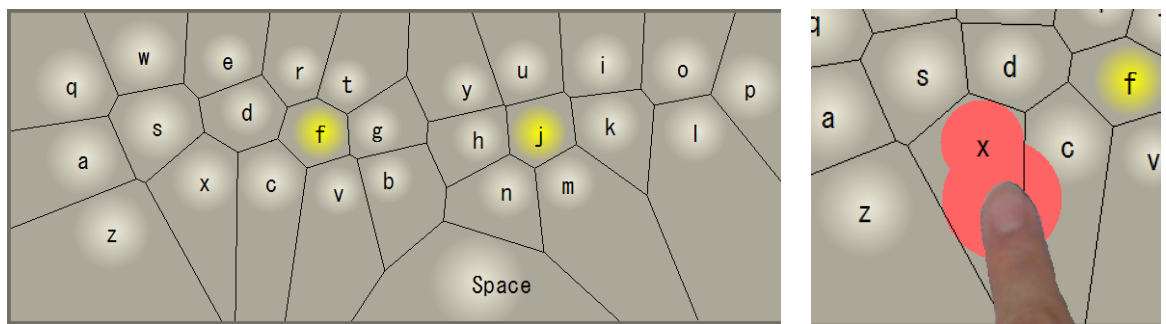

Fig. 3. Design of visual feedback in CATKey. Left: the overview of CATKey with lines and color circles. Right: bubble cursor-like visual feedback.

\section{Evaluation}

We evaluated the usability of CATKey based on the ISO 9241-4 [4]. We measured the entry speed and error rate using a hardware Qwerty keyboard, a software Qwerty keyboard, and CATKey, and assessed the comfort; finally, we conducted a debriefing interview.

Ten college students (two female and eight male) participated in the evaluation. They all use a hardware Qwerty keyboard regularly, but seldom use a touchscreen keyboard for their daily activities.

\section{A CAD Center NEXTRAX 15} touchscreen computer is used. It has a 15inch TFT monitor with $1024 \times 768$-pixel resolution. CATKey is implemented using C\# of Microsoft Corporation's Visual Studio.Net 2003. Figure 4 shows a user typing with NEXTRAX 15, which has 20 degree mounting angle. For hardware keyboards, Dell Computer Corporation's standard keyboard was used. The sentences were chosen randomly from

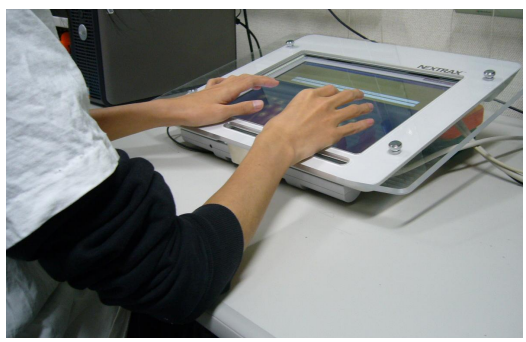

Fig. 4. Experimental setting (NEXTRAX 15) among pangrams such as "a quick brown fox jumps over the lazy dog," "how quickly daft jumping zebras vex," and "pack my box with five dozen liquor jugs."

Each experimental session included ten sentences for the typing task. The participant performed two practice sessions, then completed four sessions for measurement. The presentation order of keyboard type was counterbalanced. 
Results indicate that the respective mean entry speeds of the hardware Qwerty keyboard, software Qwerty keyboard, and CATKey are 33.5, 23.8, and $22.8 \mathrm{wpm}$. A significant difference exists among the keyboards $(F(2,18)=7.5, p<0.05)$; but no significant difference is apparent between the levels of the software Qwerty keyboard and CATKey. Similarly, the mean error rate of the hardware Qwerty keyboard, software Qwerty keyboard, and CATKey are respectively 3.4, 5.9, and 6.8\%. A significant difference exists among the keyboards $(F(2,18)=6.3(p<0.05))$; but no significant difference was found between the levels of software Qwerty keyboard and CATKey. In addition, no significant difference exists on the assessment of comfort between the software Qwerty keyboard and CATKey.

\section{Conclusion and Future Work}

In this paper, we described the design of CATKey: a customizable and adaptable touchscreen keyboard with the bubble cursor-like visual feedback. Early evaluation showed that the mean entry speed and mean input error rate of CATKey have no statistical difference from that of a standard software Qwerty keyboard. During the debriefing interview, however, the participants expressed their preference for the design and usability of CATKey. We will further investigate the usability of CATKey and explore various key arrangements such as the Metropolis keyboard [9].

\section{References}

1. Benali-Khoudja, M., Hafez, M., Alexandre, J.M., Kheddar, A.: Tactile Interfaces: A Stateof-the-art Survey. In: Proc. ISR, pp. 721-726 (2004)

2. Grossman, T., Balakrishnan, R.: The Bubble Cursor: Enhancing target acquisition by dynamic resizing of the cursor's activation area. In: Proc. CHI 2005, pp. 281-290 (2005)

3. Himberg, J., Hakkila, J., Kangas, P., Mantyjarvi, J.: On-line personalization of a touch screen based keyboard. In: Proc. IUI 2003, pp. 77-84 (2003)

4. ISO9241-4. Ergonomic requirements for office work with visual display terminals (VDTs), Part 4: Keyboard requirements (1998)

5. MacKenzie, I.S., Zhang, S.X., Soukoreff, R.W.: Text entry using soft keyboards. BIT 18, 235-244 (1995)

6. Shneiderman, B.: Touch Screens Now Offer Compelling Uses. IEEE Software, 93-94 (March 1991)

7. Sears, A., Revis, D., Swatski, J., Crittenden, R., Shneiderman, B.: Investigating touchscreen typing: The effect of keyboard size on typing speed. BIT 12(1), 17-22 (1993)

8. Sears, A., Shneiderman, B.: High-precision touchscreens: design strategies and comparisons with a mouse. Intl. J. of Man-Machine Studies 34(4), 593-613 (1991)

9. Zhai, S., Hunter, M., Smith, B.A.: The Metropolis Keyboard: An Exploration of Quantitative Techniques for Virtual Keyboard Design. In: Proc. UIST 2000, pp. 119-128 (2000) 